BMJ Open

Diabetes

Research

\& Care

\section{Association between variability in body mass index and development of type 2 diabetes: Panasonic cohort study}

Hiroshi Okada (D) , ${ }^{1}$ Masahide Hamaguchi (D) , ${ }^{2}$ Momoko Habu, ${ }^{1}$ Kazushiro Kurogi, ${ }^{3}$ Hiroaki Murata, ${ }^{4}$ Masato Ito, ${ }^{3}$ Michiaki Fukui ${ }^{2}$
To cite: Okada $\mathrm{H}$, Hamaguchi M, Habu M, et al. Association between variability in body mass index and development of type 2 diabetes: Panasonic cohort study. BMJ Open Diab Res Care 2021;9:e002123. doi:10.1136/ bmjdrc-2021-002123

Received 9 January 2021 Revised 26 February 2021 Accepted 28 March 2021
Check for updates

C Author(s) (or their employer(s)) 2021. Re-use permitted under CC BY-NC. No commercial re-use. See rights and permissions. Published by BMJ.

${ }^{1}$ Diabetes and Endocrinology, Matsushita Memorial Hospital, Moriguchi, Japan

${ }^{2}$ Endocrinology and Metabolism, Kyoto Prefectural University of Medicine, Kyoto, Japan

${ }^{3}$ Health Care Center, Panasonic Health Insurance Organization, Moriguchi, Japan

${ }^{4}$ Orthopaedic Surgery,

Matsushita Memorial Hospital, Moriguchi, Japan

Correspondence to Dr Hiroshi Okada;

continental4x33211@msn.com

\section{ABSTRACT}

Introduction Contrasting results have been reported for the association between the variability in body weight and development of diabetes. In the present study, we evaluated the association between the variability in body mass index (BMI) and development of type 2 diabetes in 19412 Japanese participants without obesity and without body weight gain or loss during the study period.

Research design and methods We recorded body weight of the participants consecutively each year in Panasonic Corporation, Osaka, Japan from 2008 to 2014 to evaluate the variability of BMI. The participants with obesity (BMI $\geq 25 \mathrm{~kg} / \mathrm{m}^{2}$ ) at baseline and body weight gain or loss from 2008 to 2014 (delta BMI $\geq \pm 1 \mathrm{~kg} / \mathrm{m}^{2}$ ) were excluded from the study. In total, 416 participants developed type 2 diabetes from 2015 to 2018. We used coefficient of variation (CV) to represent the variability in BMI during 6 years of the study period.

Results Cox regression analyses revealed that the risk of developing type 2 diabetes was higher in the fourth quartile (HR 1.33; $95 \% \mathrm{Cl} 1.01$ to 1.75) of CV of BMI than that in the first quartile (lowest quartile) of CV of BMl after adjusting for multiple confounding factors. The risk for developing diabetes increased by $11.1 \%$ per $1 \%$ increase in CV of BMI.

Conclusions In conclusion, the variability in BMI is a risk factor for the development of diabetes in the Japanese population without obesity and without body weight gain or loss.

\section{INTRODUCTION}

In recent years, the prevalence of diabetes has increased globally and the medical costs have shown a similar trend. Therefore, the prevention of the development of diabetes is a common concern in clinical care settings. It has been well established that several risk factors including obesity accelerate the development of diabetes. Several studies have investigated the association between variability in body weight and development of diabetes. ${ }^{1-7}$ Some studies have reported that the presence of obesity and variability in body weight is a risk factor for the development of diabetes. ${ }^{367}$ In contrast, several studies have suggested that instead of variability in body weight, baseline body mass index (BMI) is

\section{Significance of this study}

What is already known about this subject?

- Obesity or body weight gain accelerate the development of diabetes.

- The association between the variability in body weight and development of diabetes is controversial.

What are the new findings?

- The risk for developing diabetes increased by $11.1 \%$ per $1 \%$ increase in coefficient of variation of body mass index (BMI).

- The risk for developing diabetes increased by $15.0 \%$ per $1 \%$ increase in coefficient of variation of BMI in participants without impaired fasting glucose at baseline.

- The variability in BMI is a risk factor for the development of diabetes in population without obesity and without body weight gain or loss.

How might these results change the focus of research or clinical practice?

- The variability in $\mathrm{BMl}$ is noteworthy to reduce the risk of the development of diabetes in population without obesity and without weight gain or loss.

a strong risk factor for the development of diabetes. ${ }^{145}$ The association between the variability in body weight and development of diabetes is controversial. One of the underlying reasons for contrasting results among the studies is the difference in definition of the variability in body weight. Other underlying reasons may be the differences in the duration of study, cohort size and/or accuracy of recording body weight. Moreover, we consider two significant reasons for the contrasting associations reported between variability in body weight and development of diabetes. First, the presence of obesity at baseline and body weight gain during the study period can lead to the development of diabetes. Body weight loss during study period can prevent for the development of diabetes. Although body weight at baseline and body weight gain or loss during the study period 
can affect the development of diabetes, many studies have not elucidated these effects. Second, the difference in race can influence the development of diabetes. The BMI of Japanese patients with diabetes is similar to that of the Japanese population as a whole. ${ }^{8}$ Variability in body weight may be more significant than the body weight at baseline in the Japanese population without obesity.

To the best of our knowledge, no study has elucidated the association between the variability in body weight and the development of type 2 diabetes in a population without obesity and without body weight gain or loss during the study period. Therefore, the present study, for the first time, investigated the association between the variability in BMI and the development of type 2 diabetes in the Japanese population without obesity and without body weight gain or loss during the study period.

\section{MATERIALS AND METHODS}

\section{Study design and data collection}

The present long-term retrospective cohort study comprised participants from a medical health check-up program, which was conducted in Panasonic Corporation, Osaka, Japan. The purpose of this program was to promote public health through the early detection of chronic diseases including metabolic disorders and the evaluation of their underlying risk factors. All employees participate in this program every year. The employer shall, as provided for by the Ordinance of the Ministry of Health, Labor and Welfare, have medical examinations of workers conducted by a physician. The present study used the data collected between 2008 and
2018, which were retrieved from the database named Panasonic cohort study.

The blood samples were collected after $>10$ hours of fasting. Body weight and height were recorded using an automatic weight and height machine. The participants wore only underwear when their body weight and height were recorded. The baseline characteristics were assessed using the self-administered questionnaire, which was standardized and has been previously validated. Forty-one items were included in the questionnaire. The participants were classified as non-smokers, past smokers and current smokers. Eating speed was classified as fast, normal and slow. The participants were asked about their habit of breakfast. The participants who consumed alcohol daily were classified as alcohol drinkers. The participants who played any sport twice a week regularly were classified as regular exercisers. Type 2 diabetes was defined by fasting plasma glucose level $\geq 126 \mathrm{mg} / \mathrm{dL}$, having a self-reported history of diabetes and/or the use of antidiabetic medication. Impaired fasting glucose (IFG) was defined by the fasting plasma glucose level of $110-125 \mathrm{mg} / \mathrm{dL}$. The variability in BMI was assessed from 2008 to 2014. The development of type 2 diabetes was assessed every year from 2015 to 2018.

\section{Exclusion criteria}

In total, 140590 employees underwent a medical health check-up in 2008. The participants who did not undergo health check-up consecutively from 2008 to 2018 were excluded $(n=65256)$ from the study. The participants

Table 1 Characteristics of participants at baseline

\begin{tabular}{|c|c|c|c|c|}
\hline & All & Development of diabetes (-) & Development of diabetes (+) & $P$ value \\
\hline $\mathrm{N}$ & 19412 & 18996 & 416 & - \\
\hline Age (years) & $42.16(6.05)$ & $42.1(6.06)$ & $44.8(4.97)$ & $<0.0001$ \\
\hline Sex (male/female) & 15 909/3503 & 15 513/3483 & $396 / 20$ & $<0.0001$ \\
\hline BMl at baseline $\left(\mathrm{kg} / \mathrm{m}^{2}\right)$ & $21.54(2.04)$ & $21.52(2.04)$ & $22.50(1.81)$ & $<0.0001$ \\
\hline SBP $(\mathrm{mm} \mathrm{Hg})$ & $116.11(13.16)$ & $115.98(13.1)$ & $121.63(14.50)$ & $<0.0001$ \\
\hline $\mathrm{DBP}(\mathrm{mm} \mathrm{Hg})$ & $72.49(10.15)$ & $72.39(10.12)$ & $76.84(10.55)$ & $<0.0001$ \\
\hline LDL cholesterol (mg/dL) & $119.58(29.93)$ & $119.36(29.758)$ & $129.72(34.64)$ & $<0.0001$ \\
\hline HDL cholesterol (mg/dL) & $62.19(15.13)$ & $62.3(15.14)$ & $57.43(14.18)$ & $<0.0001$ \\
\hline Triglycerides (mg/dL) & $101.13(76.79)$ & $100.5(76.58)$ & $131.94(80.08)$ & $<0.0001$ \\
\hline Glucose (mg/dL) & $91.57(8.17)$ & $91.30(7.93)$ & $103.65(9.762)$ & $<0.0001$ \\
\hline Uric acid (mg/dL) & $5.69(1.33)$ & $5.68(1.33)$ & $6.21(1.39)$ & $<0.0001$ \\
\hline Smoking (none/past/current) & 10 363/2579/6470 & 10 205/2530/6261 & $158 / 49 / 209$ & $<0.0001$ \\
\hline Eating speed (fast/normal/slow) & $5660 / 12163 / 1589$ & $5504 / 11932 / 1570$ & $156 / 241 / 19$ & $<0.0001$ \\
\hline Skipping breakfast (+/-) & $4133 / 15279$ & 4018/14 978 & $115 / 301$ & 0.001 \\
\hline Alcohol drinker (+/-) & $5192 / 14220$ & $5039 / 13957$ & $153 / 263$ & $<0.0001$ \\
\hline Physical exercise (+/-) & $2958 / 16454$ & 2889/16 107 & $69 / 347$ & 0.44 \\
\hline CV of BMI (\%) & $2.22(0.96)$ & $2.21(0.96)$ & $2.30(1.04)$ & 0.037 \\
\hline
\end{tabular}

Data are presented as mean (SD) or absolute number.

BMI, body mass index; CV, coefficient of variation; DBP, diastolic blood pressure; HDL, high-density lipoprotein; LDL, low-density lipoprotein; SBP, systolic blood pressure. 
with diabetes at baseline and the participants who developed diabetes during the data collection for variability in BMI were excluded $(n=5614)$ from the study. We excluded participants with obesity at baseline and with body weight gain or loss from 2008 to 2014 . We defined body weight gain or loss as an increase or decrease of $>1$ $\mathrm{kg} / \mathrm{m}^{2}$ of BMI based on previous studies. ${ }^{9-11}$ The participants with obesity (BMI $\geq 25 \mathrm{~kg} / \mathrm{m}^{2}$ ) at baseline and body weight gain or loss from 2008 to 2014 (delta BMI $\geq \pm 1 \mathrm{~kg}$ / $\left.\mathrm{m}^{2}\right)$ were excluded $(\mathrm{n}=26321)$ from the study. The participants with missing data were also excluded $(n=23987)$.

\section{Variability in BMI}

We collected BMI data consecutively from 2008 to 2014 to evaluate the variability in BMI. We calculated the coefficient of variation (CV) of BMI to assess the variability of BMI from 2008 to 2014. The CV was calculated as follows: $\mathrm{CV}=\mathrm{SD} \times 100 /$ average BMI. ${ }^{12}$ Because we need to consider the effect of change in BMI after data collection of CV of BMI, we assessed change in BMI from 2015 to the end of the study. We defined change in BMI after data collection as the difference between BMI at the end of the study and that in 2015.

\section{Statistical analyses}

The means and frequencies of potential confounding variables were calculated. The differences in general characteristics at baseline according to the development of diabetes at follow-up were assessed by the t-test and $\chi^{2}$ test as appropriate. The CV of BMI were divided into quartiles: $<1.56,1.56-2.03,2.04-2.65$ and $>2.65 \%$ in all participants or $<1.56,1.56-2.03,2.04-2.66$ and $>2.67 \%$ in the participants without IFG at baseline. The association between the variability in BMI and the development of type 2 diabetes was evaluated by Cox regression analyses using multivariate models. Quartiles of CV of BMI and continuous variable of CV of BMI were added in model 1 and model 2, respectively. Covariates included in the multivariate model are the factors associated with the development of type 2 diabetes. Multivariate model was adjusted for age, sex, BMI at baseline, change in BMI after data collection, systolic blood pressure (SBP), levels of low-density lipoprotein (LDL) cholesterol, high-density lipoprotein (HDL) cholesterol, triglycerides, glucose at baseline, smoking status, eating speed, skipping breakfast, alcohol consumption and physical exercise. We also assessed the association between the variability in BMI and the development of type 2 diabetes in patients without IFG at baseline who were likely to develop diabetes. All continuous variables are presented as mean $\pm \mathrm{SD}$ or absolute number. The differences with $\mathrm{p}$ value $<0.05$ were considered statistically significant. The associations are presented as HRs with 95\% CI. The statistical analyses were performed using JMP software, V.10 (SAS Institute, Cary, North Carolina, USA).

\section{RESULTS}

The baseline characteristics of the participants enrolled in this study are shown in table 1 . Table 2 shows the baseline characteristics of the participants without IFG at baseline. In total, 416 participants among all participants

Table 2 Characteristics of the participants without impaired fasting glucose at baseline

\begin{tabular}{|c|c|c|c|c|}
\hline & All & Development of diabetes (-) & Development of diabetes (+) & $P$ value \\
\hline $\mathrm{N}$ & 19031 & 18716 & 315 & - \\
\hline Age (years) & $42.11(6.06)$ & $42.07(6.06)$ & $44.54(5.14)$ & $<0.0001$ \\
\hline BMl at baseline $\left(\mathrm{kg} / \mathrm{m}^{2}\right)$ & $21.53(2.04)$ & $21.52(2.04)$ & $22.56(1.79)$ & $<0.0001$ \\
\hline SBP (mm Hg) & $115.92(13.06)$ & $115.84(13.02)$ & $120.76(14.40)$ & $<0.0001$ \\
\hline LDL cholesterol (mg/dL) & $119.45(29.85)$ & $119.28(29.73)$ & $129.21(35.17)$ & $<0.0001$ \\
\hline HDL cholesterol (mg/dL) & $62.23(15.13)$ & $62.30(15.14)$ & $57.70(14.21)$ & $<0.0001$ \\
\hline Triglycerides (mg/dL) & $100.36(73.45)$ & $99.83(73.26)$ & 131.73 (78.32) & $<0.0001$ \\
\hline Glucose (mg/dL) & $91.10(7.53)$ & $90.96(7.45)$ & $99.77(7.45)$ & $<0.0001$ \\
\hline Uric acid (mg/dL) & $5.68(1.33)$ & $5.67(1.33)$ & $6.21(1.37)$ & $<0.0001$ \\
\hline Alcohol drinker (+/-) & $5040 / 13991$ & $4928 / 13788$ & $112 / 203$ & 0.002 \\
\hline Physical exercise (+/-) & 2892/16 139 & $2838 / 15878$ & $54 / 261$ & 0.34 \\
\hline CV of BMI (\%) & $2.22(0.96)$ & $2.21(0.96)$ & $2.35(1.08)$ & 0.016 \\
\hline
\end{tabular}

Data are presented as mean (SD) or absolute number.

BMI, body mass index; CV, coefficient of variation; DBP, diastolic blood pressure; HDL, high-density lipoprotein; LDL, low-density lipoprotein; SBP, systolic blood pressure. 
Table 3 Multivariate adjusted HRs for development of diabetes in all participants

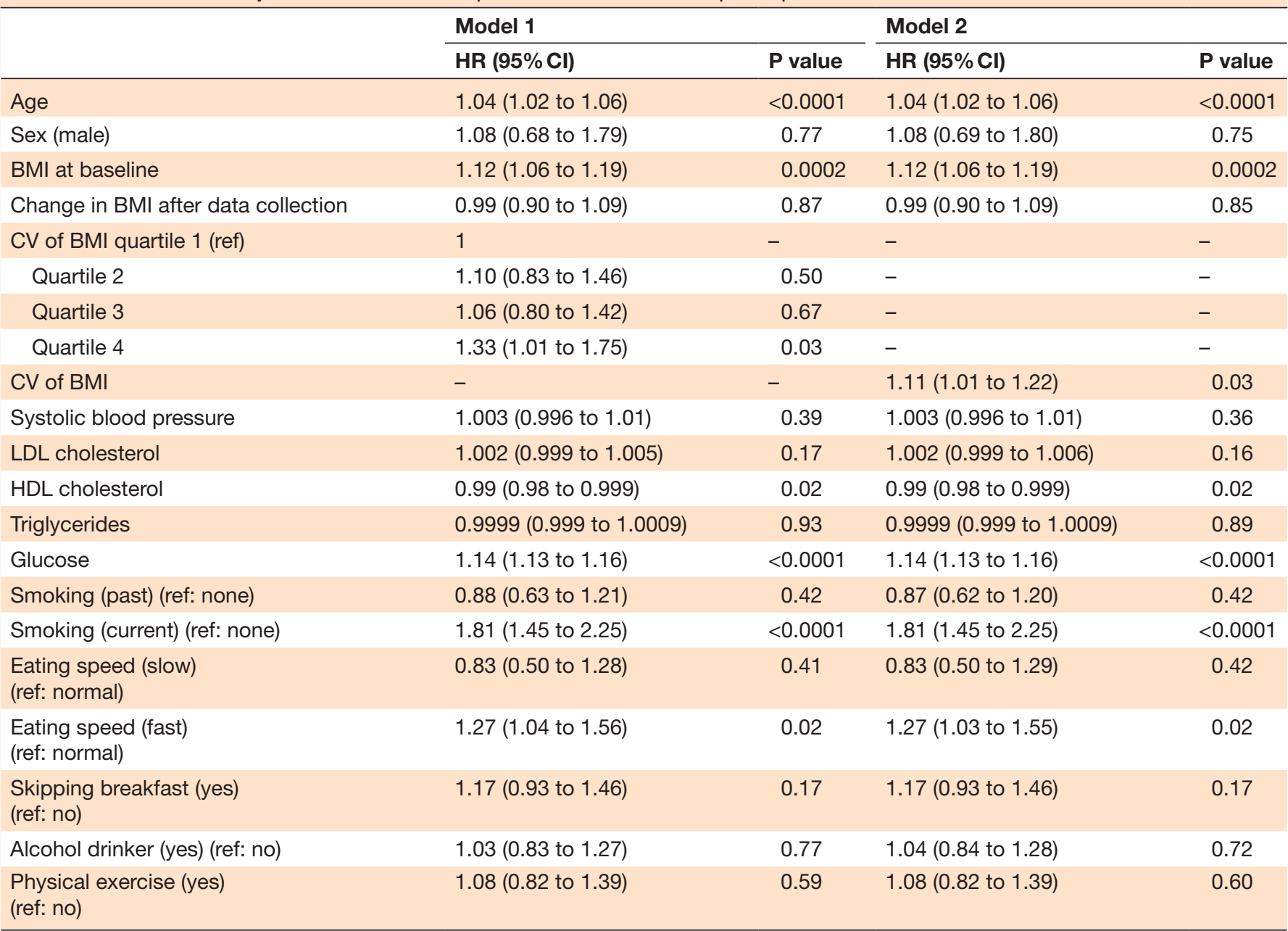

BMI, body mass index; CV, coefficient of variation; HDL, high-density lipoprotein; LDL, low-density lipoprotein; ref, reference.

(315 participants among those without IFG at baseline) developed type 2 diabetes from 2015 to 2018.

Tables 3 and 4 show the adjusted HRs in multivariate models for the development of diabetes in all participants or in the participants without IFG at baseline. The risk of developing type 2 diabetes was higher in the fourth quartile of CV of BMI than that in the lowest quartile (first quartile) of CV of BMI in all participants and in those without IFG at baseline (model 1; HR 1.33; 95\% CI 1.01 to 1.75 and HR $1.51 ; 95 \%$ CI 1.10 to 2.08 , respectively). The risk for developing diabetes increased by $11.1 \%$ and $15.0 \%$ per $1 \%$ increase in $\mathrm{CV}$ of BMI in all participants and in those without IFG at baseline, respectively (model 2). Age, BMI at baseline, HDL cholesterol, glucose, current smoking habit and fast eaters were also associated with the increased odds of developing type 2 diabetes in all participants. Age, BMI at baseline, glucose and current smoking habit were associated with the increased odds of developing type 2 diabetes in the participants without IFG at baseline. If we change the combination of period for data collection of $\mathrm{CV}$ of BMI and follow-up as follows: 2008-2012 and 2013-2018, 2008-2013 and 2014-2018 or 2008-2015 and 2016-2018 respectively, the HR per
$1 \%$ increase in CV of BMI was 1.04 (95\% CI 0.93 to 1.15 , $\mathrm{p}=0.50), 1.13(95 \%$ CI 1.03 to $1.24, \mathrm{p}=0.013)$ or 1.15 (95\% CI 1.03 to $1.37, \mathrm{p}=0.0086$ ) in all participants.

\section{DISCUSSION}

The CV of BMI was associated with the increased odds of the development of type 2 diabetes. The main results were identified if the participants with IFG at baseline were excluded. The major finding of the present study is that the variability in BMI is an independent risk factor for the development of type 2 diabetes in a Japanese population. When CV of BMI and the development of type 2 diabetes were assessed from 2008 to 2012 and from 2013 to 2018, we found no association between CV of BMI and the development of type 2 diabetes because the period for data collection for CV of BMI might be short.

The association between the variability in body weight and the development of diabetes is still controversial. Although many studies have evaluated the effect of body weight or BMI at baseline on the development of diabetes, few studies have evaluated the effect of body weight gain or loss. A study evaluated the association 
Table 4 Multivariate adjusted HRs for the development of diabetes in the participants without impaired fasting glucose at baseline

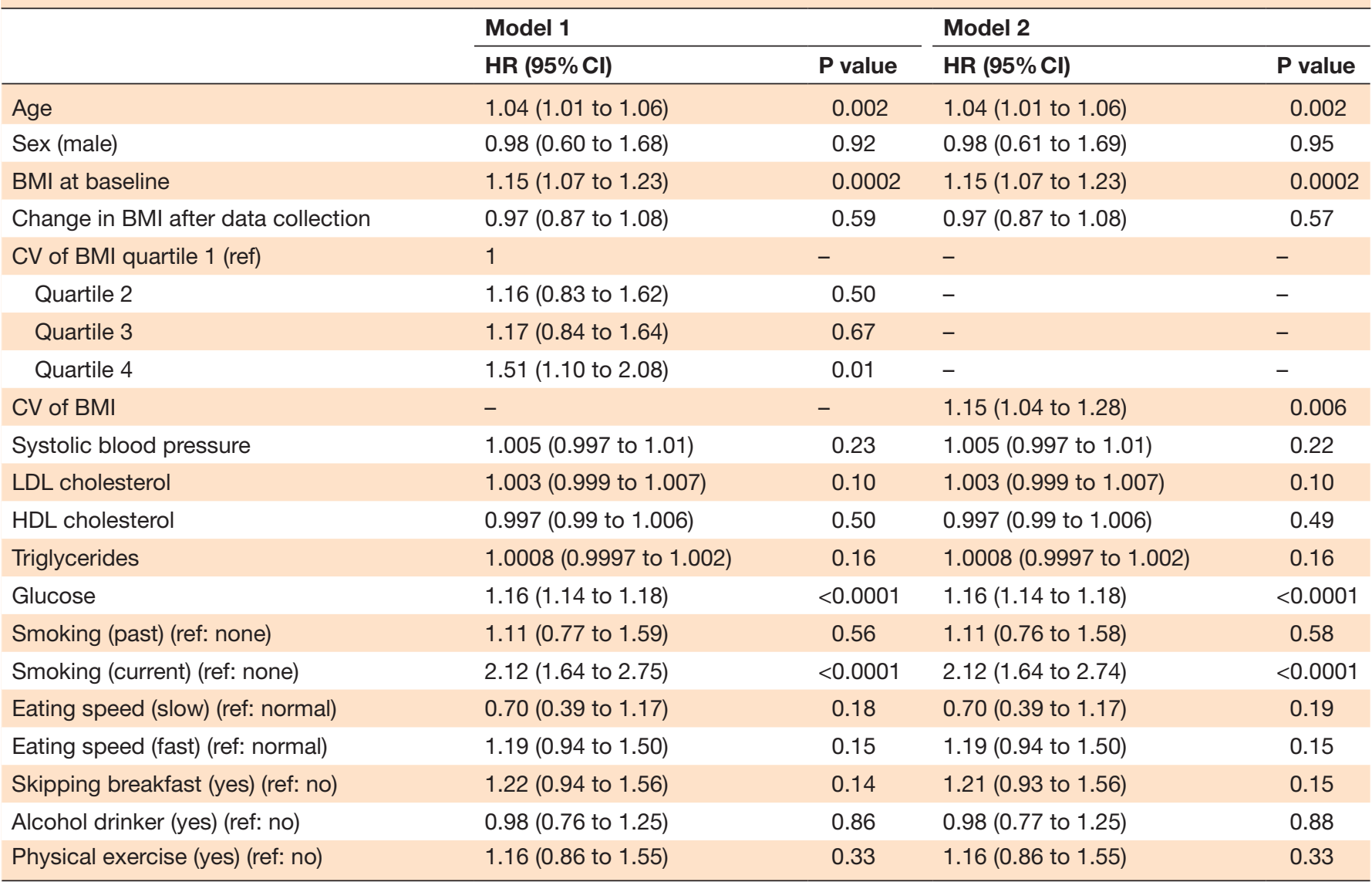

BMI, body mass index; CV, coefficient of variation; HDL, high-density lipoprotein; LDL, low-density lipoprotein; ref, reference.

between variability in body weight and body weight gain or loss and reported the association between the variability in body weight and the development of diabetes. ${ }^{13}$ However, the duration of this study was very short and the variability in body weight was calculated for the data of only 2-3 years. ${ }^{13}$ The authors defined the weight-stable group with the change in body weight $< \pm 5 \% .{ }^{13}$ However, we consider that the $\pm 5 \%$ change in body weight during 2-3 years was large and unstable. Therefore, the exact effect of body weight gain or loss was not assessed. In the present analysis, we excluded the participants with obesity (BMI $\geq 25 \mathrm{~kg} / \mathrm{m}^{2}$ ) at baseline and body weight gain or loss during 6 years period (delta BMI $\geq \pm 1 \mathrm{~kg} / \mathrm{m}^{2}$ ). Body weight was recorded in accuracy using an automatic weight and height machine in this study. Moreover, we have added change in BMI after data collection of the variability of BMI to multivariate models in order to exclude the effect of change in BMI after data collection of the variability of BMI.

It is unclear how the variability in body weight causes the development of diabetes. There were several reports which assess the association between the variability in body weight and glucose tolerance. A study reported the association between the variability in body weight and fatty acid metabolism. ${ }^{14}$ It was found that the variability in body weight was associated with an increase in the size of adipocytes and the amount of lipogenic enzymes, including fatty acid synthase, acetyl-CoA carboxylase, malic enzyme, pyruvate kinase and lipoprotein lipase in an animal model. ${ }^{14}$ Additionally, it has been reported that the variability in body weight is associated with the increase in the amounts of myristic acid, palmitic acid, palmitoleic acid and stearic acid, which leads to the impairment in glucose metabolism. ${ }^{15}$ In contrast, the variability in body weight has been associated with the decrease in the amounts of linoleic acid and $\alpha$-linolenic acid, which leads to the improvement in glucose metabolism. ${ }^{16} 17$ Tamakoshi et $a l^{18}$ have reported that the variability in body weight is associated with elevated $\mathrm{C}$ reactive protein among Japanese population. Elevated $\mathrm{C}$ reactive protein is known as the predictor of the development of diabetes. ${ }^{19}$ Chronic inflammation, which is caused by variability in body weight, might be associated with the development of diabetes. We should also consider the differences among races while accounting for the association between variability in body weight and the development of diabetes. The prevalence of obesity is lower in Asian populations than in the Western populations. The BMI of Japanese patients with diabetes is similar to that in the general Japanese population. ${ }^{8}$ This characteristic finding is important to be considered when evaluating the association between the variability in body weight 
and the development of diabetes. Asian populations may be more susceptible to variations in body weight than Western populations. It has been reported that the variability in body weight can cause hyperinsulinemia. ${ }^{20}$ The disturbance of insulin secretion, which is characteristic in Asian patients with diabetes, might be related to vulnerability to variability in body weight.

The strengths of our study are long study duration and large sample size. Moreover, the data of body weight are accurate. However, this study has several limitations. First, hemoglobin A1c (HbAlc) level was not included for the diagnosis of diabetes. Because we have no data on HbAlc, however a previous study had reported that the correlation coefficient between fasting glucose level and HbAlc level was $0.85,{ }^{21}$ we considered that it was acceptable to assess the development of diabetes through fasting glucose levels and a self-reported history of diabetes. Second, the use of diuretics and psychoactive drugs could affect the variability in BMI. Unfortunately, however, we have no data about the use of diuretics and psychoactive drugs. Third, the study population consisted of Japanese men and women and they are relatively young. Therefore, it is uncertain whether the findings of this study are generalized to other ethnic groups and other generations.

It is important to focus on the variability in BMI in the Japanese population without obesity and without body weight gain or loss in clinical scene to reduce the risk of the development of diabetes. In conclusion, the variability in BMI is a risk factor for the development of diabetes in the Japanese population.

Contributors All persons who meet authorship criteria are listed as authors, and all authors certify that they have participated sufficiently in the work to take public responsibility for the content. $\mathrm{HO}$ researched data and wrote manuscript. MaH, $\mathrm{MoH}, \mathrm{KK}$ and $\mathrm{HM}$ contributed to discussion. MI researched data and contributed to discussion. MF reviewed and edited the manuscript.

Funding The authors have not declared a specific grant for this research from any funding agency in the public, commercial or not-for-profit sectors.

Competing interests $\mathrm{HO}$ has received honoraria from Kissei Pharmaceutical, Daiichi Sankyo, Sanofi, Mitsubishi Tanabe Pharma, Takeda Pharmaceutical, Kyowa Kirin, MSD, Sumitomo Dainippon Pharma, Novo Nordisk Pharma, Ono Pharmaceutical and Eli Lilly Japan. MaH has received grant support from Asahi Kasei Pharma, Mitsubishi Tanabe Pharma, Nippon Boehringer Ingelheim, Sanofi, Daiichi Sankyo, Takeda Pharmaceutical, Kyowa Kirin, Sumitomo Dainippon Pharma, Astellas Pharma, Novo Nordisk Pharma and Eli Lilly Japan. MF has received grants from Nippon Boehringer Ingelheim, Kissei Pharmaceutical, Mitsubishi Tanabe Pharma, Daiichi Sankyo, Takeda Pharmaceutical, Sanofi, Astellas Pharma, MSD, Kyowa Hakko Kirin, Sumitomo Dainippon Pharma, Novo Nordisk Pharma, Kowa Pharmaceutical, Eli Lilly Japan, Sanwa Kagaku Kenkyusho, Taisho Pharmaceutical, Ono Pharmaceutical, Terumo, Teijin Pharma, Nippon Chemiphar and Johnson \& Johnson and received honoraria from Nippon Boehringer Ingelheim, Kissei Pharmaceutical, Daiichi Sankyo, Sanofi, Mitsubishi Tanabe Pharma, Takeda Pharmaceutical, Astellas Pharma, Kyowa Kirin, MSD, Sumitomo Dainippon Pharma Kowa Pharmaceutical, Novo Nordisk Pharma, Ono Pharmaceutical, Sanwa Kagaku Kenkyusho, Eli Lilly Japan, Taisho Pharmaceutical, Bayer Yakuhin, AstraZeneca, Mochida Pharmaceutical and Combi.

Patient consent for publication Not required.

Ethics approval This study was approved by the local ethics committee of Panasonic Health Insurance Organization and was conducted in accordance with the principles of Declaration of Helsinki.

Provenance and peer review Not commissioned; externally peer reviewed.
Data availability statement Data are available on reasonable request. The datasets used and/or analyzed during the current study are available from the corresponding author on reasonable request.

Open access This is an open access article distributed in accordance with the Creative Commons Attribution Non Commercial (CC BY-NC 4.0) license, which permits others to distribute, remix, adapt, build upon this work non-commercially, and license their derivative works on different terms, provided the original work is properly cited, appropriate credit is given, any changes made indicated, and the use is non-commercial. See: http://creativecommons.org/licenses/by-nc/4.0/.

\section{ORCID iDs}

Hiroshi Okada http://orcid.org/0000-0002-1707-970X

Masahide Hamaguchi http://orcid.org/0000-0002-8651-4445

\section{REFERENCES}

1 Oh TJ, Moon JH, Choi SH, et al. Body-Weight fluctuation and incident diabetes mellitus, cardiovascular disease, and mortality: a 16-year prospective cohort study. J Clin Endocrinol Metab 2019;104:639-46.

2 Yokomichi H, Ohde S, Takahashi O, et al. Weight cycling and the subsequent onset of type 2 diabetes mellitus: 10-year cohort studies in urban and rural Japan. BMJ Open 2017;7:e014684.

3 Rhee E-J, Cho JH, Kwon H, et al. Increased risk of diabetes development in individuals with weight cycling over 4 years: The Kangbuk Samsung Health study. Diabetes Res Clin Pract 2018;139:230-8.

4 Waring ME, Eaton CB, Lasater TM, et al. Incident diabetes in relation to weight patterns during middle age. Am J Epidemiol 2010;171:550-6.

5 Field AE, Manson JE, Laird N, et al. Weight cycling and the risk of developing type 2 diabetes among adult women in the United States. Obes Res 2004:12:267-74.

6 Neamat-Allah J, Barrdahl M, Hüsing A, et al. Weight cycling and the risk of type 2 diabetes in the EPIC-Germany cohort. Diabetologia 2015;58:2718-25.

7 French SA, Folsom AR, Jeffery RW, et al. Weight variability and incident disease in older women: the lowa women's health study. Int J Obes Relat Metab Disord 1997:21:217-23.

8 Sone $\mathrm{H}$, Mizuno S, Ohashi Y, et al. Type 2 diabetes prevalence in Asian subjects. Diabetes Care 2004;27:1251-2.

9 Wing RR, Lang W, Wadden TA, et al. Benefits of modest weight loss in improving cardiovascular risk factors in overweight and obese individuals with type 2 diabetes. Diabetes Care 2011;34:1481-6.

10 Kodama S, Horikawa C, Yoshizawa S, et al. Body weight change and type 2 diabetes. Epidemiology 2013;24:778-9.

11 Penn L, White M, Lindström J, et al. Importance of weight loss maintenance and risk prediction in the prevention of type 2 diabetes: analysis of European diabetes prevention study RCT. PLOS One 2013;8:e57143.

12 Dolan E, O'Brien E. Blood pressure variability: clarity for clinical practice. Hypertension 2010;56:179-81.

13 Park K-Y, Hwang H-S, Cho K-H, et al. Body weight fluctuation as a risk factor for type 2 diabetes: results from a nationwide cohort study. J Clin Med 2019;8:950.

14 Sea MM, Fong WP, Huang Y, et al. Weight cycling-induced alteration in fatty acid metabolism. Am J Physiol Regul Integr Comp Physiol 2000;279:R1145-55

15 Eguchi K, Manabe I, Oishi-Tanaka Y, et al. Saturated fatty acid and TLR signaling link $\beta$ cell dysfunction and islet inflammation. Cell Metab 2012;15:518-33.

16 Kurotani K, Kochi T, Nanri A, et al. Plant oils were associated with low prevalence of impaired glucose metabolism in Japanese workers. PLoS One 2013;8:e64758.

17 Risérus U, Willett WC, Hu FB. Dietary fats and prevention of type 2 diabetes. Prog Lipid Res 2009;48:44-51.

18 Tamakoshi K, Yatsuya H, Kondo T, et al. Long-term body weight variability is associated with elevated C-reactive protein independent of current body mass index among Japanese men. Int $J$ Obes Relat Metab Disord 2003;27:1059-65.

19 Doi Y, Kiyohara Y, Kubo M, et al. Elevated C-reactive protein is a predictor of the development of diabetes in a general Japanese population: the Hisayama study. Diabetes Care 2005;28:2497-500.

20 Yatsuya H, Tamakoshi K, Yoshida T, et al. Association between weight fluctuation and fasting insulin concentration in Japanese men. Int J Obes Relat Metab Disord 2003;27:478-83.

21 Ito C, Maeda R, Ishida S, et al. Correlation among fasting plasma glucose, two-hour plasma glucose levels in OGTT and HbA1c. Diabetes Res Clin Pract 2000;50:225-30. 